\title{
Facilitators and barriers to adaptive implementation of the Meeting Centers Support Program (MCSP) in three European countries; the process evaluation within the MEETINGDEM study
}

\author{
L.D. Van Mierlo, ${ }^{1}$ R. Chattat, ${ }^{2}$ S. Evans, ${ }^{3}$ D. Brooker, ${ }^{3}$ F.L. Saibene, ${ }^{4}$ G. Gamberini, ${ }^{4}$ \\ E. Farina, ${ }^{4}$ C. Scorolli, ${ }^{2}$ D. Szcze' sniak, ${ }^{5}$ K.M. Urbanska, ${ }^{5}$ J. Rymaszewska, ${ }^{5}$ R.M. Dröes ${ }^{1}$ and F.J.M. Meiland ${ }^{1}$ \\ ${ }^{1}$ Department of Psychiatry, VU University Medical Centre, Amsterdam, The Netherlands \\ ${ }^{2}$ Department of Psychology, University of Bologna, Bologna, Italy \\ ${ }^{3}$ Association for Dementia Studies, University of Worcester, Henwick Grove, Worcester, UK \\ ${ }^{4}$ Santa Maria Nascente IRCCS Clinical Research Center, Don Carlo Gnocchi Foundation, Milan, Italy \\ ${ }^{5}$ Department of Psychiatry, Wroclaw Medical University, Wroclaw, Poland
}

ABSTRACT

Background: In the MEETINGDEM project, the Meeting Centers Support Program (MCSP) was adaptively implemented and evaluated in three European countries: Italy, Poland, and the United Kingdom. The aim of this study was to investigate overall and country-specific facilitators and barriers to the implementation of MCSP in these European countries.

Methods: A qualitative multiple case study design was used. Based on the theoretical model of adaptive implementation, a checklist was composed of potential facilitators and barriers to the implementation of MCSP. This checklist was administered among stakeholders involved in the implementation of MCSP to trace the experienced facilitators and barriers. Twenty-eight checklists were completed.

Results: Main similarities between countries were related to the presence of suitable staff, management, and a project manager, and the fact that the MCSP is attuned to needs and wishes of people with dementia and informal caregivers. Main differences between countries were related to: communication with potential referrers, setting up an inter-organizational collaboration network, receiving support of national organizations, having clear discharge criteria for the MCSP and continuous PR in the region.

Conclusion: The results of this study provide insight into generic and country specific factors that can influence the implementation of MCSP in different European countries. This study informs further implementation and dissemination of MCSP in Europe and may also serve as an example for the dissemination and implementation of other effective psychosocial support interventions for people with dementia and their informal caregivers across and beyond Europe.

Key words: dementia, carer, Meeting Centers Support Program, person-centered approach, implementation process, process evaluation

\section{Introduction}

There has been an increase in attention and need for personalized psychosocial interventions to support people with dementia and their informal caregivers in their needs and wishes throughout

Correspondence should be addressed to: R.M. Dröes, Department of Psychiatry, VU University Medical Center/GGZinGeest, Postbox 74077, 1070 BB, Amsterdam, The Netherlands. Phone: +31 207885454. Email: rm.droes@vumc.nl. Received 12 Jan 2017; revision requested 8 Jun 2017; revised version received 22 Aug 2017; accepted 22 Aug 2017. First published online 17 November 2017. the disease trajectory (Moniz-Cook et al., 2011). Psychosocial interventions aim to improve the quality of life of people with dementia and informal caregivers, and have shown to improve their mental health, as well as contribute to a delay in nursing home placement (Smits et al., 2007; Olazarán et al., 2010). Multi-component psychosocial support interventions that are person-centered and include practical, social, and emotional support for both the person with dementia, as well as their informal caregivers, have been proven more effective than interventions that focus only on the person 
with dementia or the informal caregiver (Brodaty et al., 2003; Smits et al., 2007). However, the dissemination and implementation of psychosocial support interventions is not always adequate in care practice and needs more attention (VernooijDassen and Moniz-Cook, 2014).

Successful implementation of combined psychosocial support often requires adaptive implementation, modified to local, regional, and country specific circumstances (Meiland et al., 2004). Implementation research that yields knowledge on specific context related facilitators and barriers of the implementation, as well as on effective adaptive implementation strategies, is therefore very important in order to embed psychosocial interventions in daily care practice and to disseminate effective interventions to other countries (Grol and Wensing, 2004; Meiland et al., 2005; Vernooij-Dassen and Moniz-Cook, 2014).

The Meeting Centers Support Program (MCSP) is an example of a proven effective multi-component support program that has been successfully implemented in the Netherlands in the last two decades (Dröes et al., 2000; Dröes et al., 2004a; Dröes et al., 2004b; Dröes et al., 2011). It is an easy accessible, socially integrated approach that aims to support community-dwelling people with mild to moderately severe dementia and their informal caregivers. The MCSP offers an integrated package of care and support that is person-centered and provides practical, social, and emotional support. This support is provided by means of a social club for the person with dementia, psychoeducational meetings and discussion groups for informal caregivers, social activities and a weekly consultation hour for both. Frequent "Meeting Centers Meetings" allow all participants, staff, and volunteers to share experiences and to influence the activities offered within the Meeting Centers.

In the European Joint Program Neurodegenerative Disease Research (JPND) funded MEETINGDEM project, MCSP was implemented in three European countries: Italy, Poland, and the United Kingdom. The aim of the MEETINGDEM project was to implement, disseminate and evaluate MCSP in these European countries (see Dröes etal. 2017) for a detailed description of the MCSP program and the design of the study).

This paper reports on the process evaluation

of the MEETINGDEM project that aimed to investigate overall and country-specific facilitators and barriers of the adaptive implementation of MCSP in the three mentioned European countries.

The process evaluation within the MEETINGDEM project consisted of two parts. The first part investigated expected facilitators and barriers of the implementation of MCSP before starting to prepare the implementation. These were compared to the actual experienced facilitators and barriers during the preparation of the implementation (Mangiaracina et al., 2017). The second part of the process evaluation was targeted on facilitators and barriers during the execution and continuation phase, and is described in this paper.

\section{Methods}

\section{Study design}

A qualitative multiple case study design was used that included the administration of checklists among stakeholders in each country who were involved in the execution and continuation phase of the implementation of MCSP in one of the three European countries. The Medical Ethics Committee of the VU University Medical Centre and the ethical committees of the three countries (Italy, Poland, and UK) approved the study protocol. In Poland this was the Bioethics Committee of the Wroclaw Medical University. In Italy this was the Ethical Committee of the Don Gnocchi Foundation. In the UK this was the Wales Research Ethics Committee.

\section{Setting and participants}

Data were collected in the period of September 2016 to October 2016 in Poland and Italy and in June 2016 in the UK. Stakeholders were selected via "purposive sampling" (Barbour, 1999) to promote qualitative rigor. Stakeholders were recruited from the Initiative Group who collaborated on the implementation of the MCSP. The Initiative Group consisted of representatives from different organizations (care and welfare) with expertise in dementia care and support or finance, and stakeholders at the local level (municipality) and at the regional/national level (e.g. Alzheimer association). Oral consent was acquired from all participants prior to participation.

\section{Theoretical model and checklist}

The process evaluation was based on the theoretical model of adaptive implementation (Dröes et al., 2003; Meiland et al., 2004). This model was developed in previous research into facilitators and barriers of implementation of MCSP in the Netherlands. (Figure 1) (Dröes et al., 2003).

This model describes external factors (e.g. characteristics of the intervention, operational preconditions, personal, and financial resources) that can influence the implementation of an intervention during various phases (preparation, execution, and continuation). It differentiates 


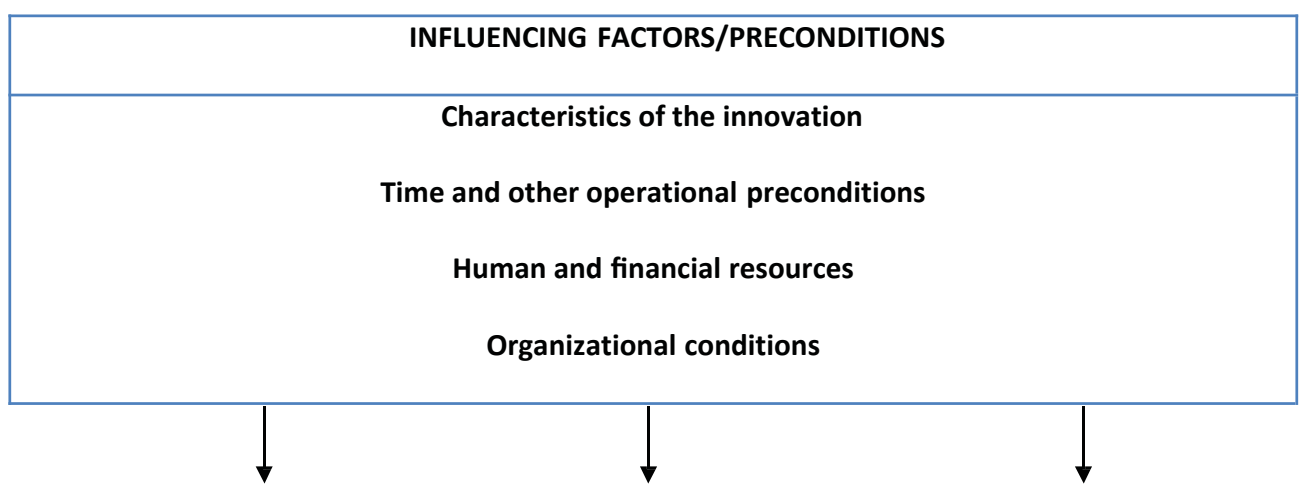

\begin{tabular}{|c|c|c|}
\hline \multicolumn{2}{|c|}{ IMPLEMENTATION PROCESS } \\
\hline $\begin{array}{c}\text { Preparation phase } \\
\text { Micro level } \\
\text { (user/primary process) }\end{array}$ & $\begin{array}{c}\text { Execution phase } \\
\text { Micro level } \\
\text { (user/primary process) }\end{array}$ & $\begin{array}{c}\text { Continuation phase } \\
\text { Micro level } \\
\text { (user/primary process) }\end{array}$ \\
$\begin{array}{c}\text { Meso level } \\
\text { (interorganizational/social } \\
\text { context) }\end{array}$ & $\begin{array}{c}\text { Meso level } \\
\text { (interorganizational/social } \\
\text { context) }\end{array}$ & $\begin{array}{c}\text { Meso level } \\
\text { (interorganizational/social } \\
\text { context) }\end{array}$ \\
$\begin{array}{c}\text { Macro level } \\
\text { (health caresystem, } \\
\text { legislation, policy) }\end{array}$ & $\begin{array}{c}\text { Macro level } \\
\text { (health caresystem, } \\
\text { legislation, policy) }\end{array}$ & $\begin{array}{c}\text { Macro level } \\
\text { (health caresystem, } \\
\text { legislation, policy) }\end{array}$ \\
\hline
\end{tabular}

Figure 1. (Colour online) Theoretical model of adaptive implementation.

between influencing factors on different levels in each of these phases: micro (user/primary process), meso (inter-organizational/social context), and macro level (healthcare system, legislation, policy) (Meiland et al., 2004; 2005).

Based on this theoretical model and lessons learned from the previous research on the adaptive implementation of MCSP in the Netherlands (Meiland et al., 2005; Practical guide on how to successfully set up a Meeting Center), stakeholders (members of the Initiative Group, who prepared the implementation of MCSP in at least one region in their country) were invited in an early stage to think about potential facilitators and barriers and about solutions for the barriers. For this, a checklist was composed of potential facilitators and barriers and translated into the language of the three participating countries (Mangiaracina et al., 2017). During this initial administration, stakeholders added several new factors that they expected to be of influence on the implementation. These additional factors were consequently added to the checklist. The completed checklist was used in the present study to investigate facilitators and barriers during the execution and continuation phase of the implementation of MCSP.

\section{Data collection}

The checklist was administered among stakeholders that agreed to participate by means of a face-to-face interview or by telephone call. Most stakeholders had also been involved in the process evaluation of the preparation phase (Mangiaracina et al., 2017). Stakeholders could indicate whether each factor had been of influence during the execution or continuation phase of the implementation and if this factor had facilitated or impeded the implementation of MCSP. A total of 28 checklists were completed. Notall factors in the checklist were administered among all stakeholders: this depended on their expertise and involvement in the implementation phases and levels.

Table 1 lists the types and number of stakeholders that were interviewed per country. In Poland a total of five types of stakeholders were interviewed, 
Table 1. Types of stakeholders that were interviewed per country

\begin{tabular}{|c|c|c|c|}
\hline $\begin{array}{l}\text { type s of } \\
\text { s takeholder }\end{array}$ & $\begin{array}{l}\text { italy } \\
(N=9)\end{array}$ & $\begin{array}{l}\text { poland } \\
(N=10)\end{array}$ & $\begin{array}{l}\text { united kingdom } \\
(N=10)\end{array}$ \\
\hline $\begin{array}{l}\text { Managers (directors, board } \\
\text { in care and/or welfare } \\
\text { setting }\end{array}$ & One Medical director & $\begin{array}{l}\text { One Director of } \\
\text { MunicipHal Social } \\
\text { Assistance Centre } \\
\text { One Director of day care } \\
\text { network }\end{array}$ & One manager of the MCSP \\
\hline $\begin{array}{l}\text { Staff in care and/or welfare } \\
\text { setting }\end{array}$ & $\begin{array}{l}\text { One MCSP coördinator } \\
\text { Two MCSP volunteers } \\
\text { One psychologist }\end{array}$ & $\begin{array}{l}\text { Two MCSP coordinators } \\
\text { One MCSP staff member } \\
\text { One day care staff } \\
\text { member }\end{array}$ & One MCSPvolunteer \\
\hline $\begin{array}{l}\text { Representatives fromlocal } \\
\text { charity/organization }\end{array}$ & & & $\begin{array}{l}\text { One representative froma } \\
\text { local care home } \\
\text { Four representatives } \\
\text { from local charitable } \\
\text { organizations relating to } \\
\text { older people }\end{array}$ \\
\hline $\begin{array}{l}\text { Representatives from } \\
\text { municipalities }\end{array}$ & $\begin{array}{l}\text { One representative from } \\
\text { the Milan Municipality }\end{array}$ & $\begin{array}{l}\text { One representative from } \\
\text { the Wroclaw } \\
\text { Municipality }\end{array}$ & \\
\hline $\begin{array}{l}\text { Representatives from } \\
\text { Health Insurance } \\
\text { Companies }\end{array}$ & $\begin{array}{l}\text { One Official of the } \\
\text { department of social } \\
\text { policy }\end{array}$ & & \\
\hline $\begin{array}{l}\text { Representatives from } \\
\text { Regional/national level }\end{array}$ & One lawyer & $\begin{array}{l}\text { One representative of a } \\
\text { non-governmental } \\
\text { organization }\end{array}$ & $\begin{array}{l}\text { Two representatives from } \\
\text { mental healthcare } \\
\text { One representative from } \\
\text { a national dementia } \\
\text { charity }\end{array}$ \\
\hline $\begin{array}{l}\text { (Representatives of) } \\
\text { Persons with } \\
\text { dementia/family } \\
\text { caregivers }\end{array}$ & One informal caregiver & $\begin{array}{l}\text { One person with dementia } \\
\text { One informal caregiver }\end{array}$ & \\
\hline
\end{tabular}

MCSP $=$ Meeting Centers Support Program.

in Italy a total of six types were interviewed, and in the UK a total of four types were interviewed. per country were integrated in a table and the

\section{Analysis}

The interviews in Poland lasted 31 minutes on average, in Italy 49 minutes on average, and in the UK 30 minutes on average.

For each potential influencing factor, it was counted how many different types of stakeholders were asked about this and experienced these as facilitators and/or barriers to the implementation. This provided us with an explorative overview of experienced facilitators and barriers to the implementation percountry.

The factors that were experienced by stakeholders during the implementation, as inventoried by the checklists, were summarized percountry for each implementation phase (execution and continuation) and organized per level (micro, meso, macro). The experienced factors and barriers 
main similarities and differences in facilitators and barriers between countries were described.

\section{Results}

Table 2 shows the results of the facilitators and barriers experienced in the three countries during the execution and continuation phase of the implementation of MCSP. The table lists both the number of stakeholders that experienced each factor as a facilitator or barrier (first number mentioned), as well as the total number of stakeholders that were asked about that factor (second number mentioned). Second, the table lists symbols, indicating whether this factor was mentioned by one type or by two or more types of stakeholders. Later, the main facilitators and barriers that were experienced by at least two different types of stakeholders in all countries are described. Next, the main differences between countries are presented. Finally, the main factors 
Were factors experienced as facilitators or

\begin{tabular}{ccc} 
poland & italy & $\begin{array}{c}\text { united } \\
\text { kingdom } \\
(N=10)\end{array}$ \\
\hline
\end{tabular}

barriers of implementation?

$\mathrm{F}+\quad \mathrm{B}-$

$\mathrm{F}+$

B -

$\mathrm{F}+$

B -

Execution phase

Microlevel

Suitability, qualification and motivation of staff/professionals/volunteers

Presence of a professional project manager

\begin{tabular}{|c|c|c|c|c|c|}
\hline $9 / 10^{a}$ & $1 / 100$ & $8 / 8$ & & $9 / 10$ & $1 / 100$ \\
\hline $10 / 10$ & & $5 / 5 x$ & & $7 / 7$ & \\
\hline $9 / 10$ & $4 / 10$ & $1 / 80$ & $7 / 8$ & $5 / 7 /$ & $2 / 7$ \\
\hline $9 / 10$ & $7 / 10$ & $3 / 6$ & $3 / 60$ & $2 / 100$ & $8 / 10$ \\
\hline $10 / 10$ & & $7 / 7$ & & $7 / 7$ & \\
\hline \multirow[t]{2}{*}{$4 / 5$} & $1 / 50$ & $2 / 4$ & $2 / 4$ & & $8 / 8$ \\
\hline & $1 / 10$ & & & & \\
\hline $6 / 7$ & $1 / 70$ & $3 / 60$ & $3 / 6$ & $4 / 5$ & $1 / 50$ \\
\hline $5 / 8$ & $3 / 8$ & & $4 / 4$ & $2 / 2$ & \\
\hline $3 / 5$ & $2 / 5$ & $3 / 4$ & $1 / 40$ & $2 / 4$ & $2 / 4$ \\
\hline $6 / 6$ & & $3 / 6$ & $3 / 6$ & & $1 / 10$ \\
\hline
\end{tabular}

Social integration possibilities at the location/in the neighborhood

Finding/including a sufficient number of patients/caregivers, presence of waiting lists

The program is attuned to needs of target population

Continuous communication with (potential) referrers and vice versa

Available time for staff to complete daily tasks

Mesolevel

Inter-organizational collaboration in the region preferably recorded in a protocol

Sound financing for the MCSP

Macrolevel

Health insurance/welfare regulations (reimbursements)

Support of national parties: Alzheimer organizations/ informal carer organizations/government

\section{Continuation phase}

Microlevel

Theoretical background of MCSP is valued (combined supportfor both person with dementia and informal caregivers)?

Integration with other activities / groups in organization

Adaption of the MCSP to needs/wishes of participants

Clear definition of the inclusion and discharge criteria

Capacity or priority in other facilities (after discharge)

Structural financing of the MCSP

Suitable and professional staff and management

Family caregivers involvement

Mesolevel

Image of MCSP as perceived by other organizations

Financial continuation of MCSP

Interregional collaboration and after discharge program (protocols)

Continuous PR in the region

Macrolevel

National law- and finance-regulations

National policies

$10 / 10$

$7 / 7$

$5 / 8$

$3 / 8$

$9 / 10$

$1 / 100$

$8 / 8$

$3 / 3$

$10 / 10 \quad 7 / 7$

$8 / 8$

$2 / 8$

$4 / 5$

$1 / 50$

$4 / 8$

$4 / 8$

/ 8

$6 / 8$

$1 / 60$

$5 / 6$

$2 / 2$

$9 / 10$

$1 / 100$

$2 / 70$

5/7

4/4

$10 / 10$

$6 / 6$

$1 / 10$

$1 / 20$

$1 / 20$

$2 / 4$

$2 / 4$

$1 / 10$

$6 / 6$

$3 / 30$

$6 / 7$

$1 / 70$

$5 / 6$

$1 / 60$

$2 / 5$

$3 / 50$

$1 / 10$

$5 / 6$

$1 / 60$

$1 / 20$

$1 / 20$

$1 / 10$

$7 / 7$

$1 / 40$

$3 / 40$

$3 / 3$

$2 / 8$

$6 / 8$

$1 / 40$

$3 / 40$

$1 / 10$

$3 / 8$

$5 / 8$

$1 / 10$

$3 / 50$

$2 / 50$

${ }^{a}$ The first number indicates the total number of stakeholders that experienced that factor as a facilitator or barrier. The second number indicates the total number of stakeholders that were asked about this factor. $=$ Factor mentioned by at least 2 types of stakeholders.

$\mathrm{O}=$ Factor mentioned by 1 type of stakeholder. 
that were considered as both a facilitator and a barrier in all countries are described.

\section{Common facilitators and barriers of the implementation in the three countries}

\author{
Execution phase
}

On a micro level, different types of stakeholders in all countries stated that qualified and motivated staff, professionals, and volunteers facilitated the implementation of MCSP. A stakeholder from the UK mentioned: "staff are key to the atmosphere and the way the Centre is run."

Overall, the presence of a professional project manager to guide the implementation was also a strong facilitator to the implementation in all countries. Last, all countries agreed that a strong facilitator of the implementation was that the MCSP program is attuned to the needs of the target population. A stakeholder from Poland: "Participants' needs help to adjust methods during activities and to get their attention and engagement. In some people there is visible betterfunctioning in daily life."

\section{Continuation phase}

On a micro level, the integration with other activities and groups at the location of the MC was experienced by different types of stakeholders in each country as clearly facilitating the implementation during the continuation phase. In Italy the people with dementia were sometimes invited by older people using the same building for activities: "Something happened in our district. Once a month the elderly from the hosting Centres meet for a pizza for example, and they invite the participants of our Centres." In Poland there was an increase in "integration between different day cares" and "integration with children from the nearest playground."

Adaption of MCSP to the needs of participants was also a strong facilitator in all three countries during the continuation phase. A stakeholder from Poland: "Adjusted activities create bigger engagement of MCSP participants, a positive atmosphere, and it helps therapists to provide care that suits the needs of participants." Finally, a clear facilitator for implementation was the continuous presence of suitable and professional staff and management.

A barrier to the implementation, that was often mentioned by different types of stakeholders in all three countries, was a problem with the capacity in other facilities that provide more advanced care for people with dementia. In Italy a stakeholder mentioned: "There is a delay in the transfer from MCSP to other facilities when more advanced care is required (for example to a day hospital or day care centres)." A stakeholder from Poland explained: "There is a long waiting time for residential care, and not enough social health care services. There is a gap in continuous support for people with dementia and their carers. Some families try to extend their time in the Meeting Centres when the person with dementia is no longer ableto participatein the programme."

On a meso level the image of MCSP as perceived by other organizations facilitated the implementation, and was mentioned by different types of stakeholders in Poland and the UK in particular. Stakeholders from Poland mentioned that "The MCSP has a positive image," and "People from other organizations are interested in learning from MCSP, how to support people with dementia and how to provide activities." A stakeholder (representative from regional/national level) from the UK said "I think it is quite positive, the MCSP is viewed well and talked about outside the area."

\section{Main differences in facilitators and barriers between countries}

Execution phase

On a micro level, the three countries experienced a difference in communication with potential referrers. In Italy, stakeholders had mixed experiences: the communication with some organizations went well: "with the healthcare organization the continuity of information was good. They have always communicated effectively about problems and they have referred people with dementia to us when there were problematic situations," but it was difficult with others: "The collaboration with other dementia organizations is growing, but this collaboration is not achieved with general practitioners and with other referrers like the hospital and the Alzheimer's unit." In Poland, most people with dementia and informal caregivers were often selfreferred. There was "good communication between families and specialists," which had a positive impact on the implementation of the MCSP. In the UK stakeholders expressed a clear problem in communication with referrers: "We need one main person to make contact with referrers, but we have no time to do this." Another stakeholder from the UK: "We need to let people know it is there, we need more publicity." As a result of this problem, the Meeting Centers in the UK had difficulties recruiting sufficient people with dementia and informal caregivers. Another stakeholder explained: "People may be worried about going to the MCSP. It is a massive step and a formal acceptance of the diagnosis. It is about trust. Getting them through 
the door is difficult." In contrast, both the Meeting Centers in Italy and Poland had to deal with waiting lists, as the demand for the MCSP exceeded the capacity. A stakeholder from Poland: "There are more people waiting and interested in MCSP participation than we can help."

On a meso level, there were differences between countries with respect to inter-organizational collaboration in the region. Stakeholders from Poland and the UKexperienced a positive impact of having a collaboration protocol for the implementation of MCSP (including information on referral to and from the MCSP, and the execution of the program). A stakeholder from Poland: "a collaboration protocol helps to form a collaboration between the MCSP and other places, it also helps in information exchange." However, stakeholders in Italy mentioned that there was support at the beginning of the project, but this gradually decreased over time, as one stakeholder concluded: "the network has not been achieved, we just received support at the beginning of the project."

On a macro level, there were differences between countries related to the support of MCSP by national organizations. Stakeholders in Italy mentioned that national associations, such as the Alzheimer's Association strongly supported the project, but collaboration with other associations was very difficult: "Some organisations were in the Initiative group, but then they did not collaborate during the execution phase." Though the advisory group (meant to support the project after the opening of the Meeting Center) was set up and held meetings initially, their collaboration decreased after 6 months and they became inactive. Stakeholders in Poland experienced clear support of national organizations (associations, local municipality, Alzheimer associations) due to an active dissemination of the MCSP. This made it possible to organize support for carers and extend the awareness about dementia in the local community. A stakeholder in the UK mentioned that there is a need for a stronger evidence base before national organizations will provide their support.

\section{Continuation phase}

On a micro level, both Italy and Poland experienced that the theoretical background of MCSP was valued and this facilitated the implementation. Though the MCSP was also valued in the UK, some stakeholders mentioned that there was a also lack of awareness about the MCSP outside of the Advisory Group and those closely connected with it: "The general public does not know it is different from day care," and: "MCSP is valued within the initiative group, but we are not sure about the wider community. The general public may think it is the same as day care." Another difference between countries was visible with respect to a clear definition of the inclusion and discharge criteria. In Italy, it was experienced that "clear criteria of inclusion are positive because they create a uniform group in which it is easier to work. The criteria were clearly defined." Also in Poland these criteria were clearly described and this facilitated the implementation of MCSP: "criteria help especially in difficult situations when the person with dementia is not able to continue in the MCSP and their families are reluctant to collaborate." However, in the UK different types of stakeholders disagreed whether the criteria were clear or not. One stakeholder mentioned: "They are becoming clearer, especially for mild to moderate dementia." The aspects that were found unclear by stakeholders in the UK related to what would happen when a MCSP would reach their maximum capacity, and there were clear concerns about what happened after a person with dementia would need more advanced care: "I worry about people when leaving and how to support the carer after a person with dementia goes into a home. Discharge is a concern and highlights there is a gap in the pathway."

The presence of structural financing for the continuation of MCSP was perceived as a facilitator to the implementation by stakeholders in Italy and Poland, but as a barrier in the UK. In Italy and Poland, the financial continuation of the Meeting Centers was expected to be supported by the Municipality. According to a MCSP program coordinator in Poland: "Social welfare, as part of the Municipality, is going to maintain the MCSP." In Italy, a stakeholder mentioned that: "The Municipality has adhered to implementation of the MCSP because they represent a perfect integration between social and health aspects." In the UK, different types of stakeholders also mentioned that it is difficult to know where to get structural funding from and that "this was a worry from the start."

On a meso level, continuous PR in the region facilitated the implementation of MCSP in Poland, in particular: "the MCSP website, open informative meetings, PR in local newspapers and radio. Also support of the MCSP leader and directors of social welfare and the Department of Social Issues in Wroclaw." This was one of the main reasons why waiting lists for the MCSP emerged. In Italy, however, a stakeholder involved in one of the MC's with no waiting list thought that there was a lack of good PR, which impeded the implementation. A stakeholder from Italy: "The awareness of MCSP is not so widespread." Another Italian stakeholder stated: "Dissemination 
should be increased. For example: the pharmacies haven't been advised of the opening of the Centres. The visibility of the project is so important," and another stakeholder: "I think Centres aren't sufficiently promoted." In the UK the MCSP was positively addressed through PR: One stakeholder mentioned: "Not sure what else could have been done. The promotional video is awesome."

\section{Factors that were experienced as both facilitators and barriers to the implementation by different stakeholders}

\section{Execution phase}

On a micro level, both facilitators and barriers were found with respect to social integration opportunities for the MCSP at the location and in the neighborhood. In Italy social integration differed across the Centers: "The situation between the two Centres was very different. In one district the integration in the neighborhood was easier than in the other district. In one district we have been welcomed, although with some difficulties. In the other district integration is still under construction. We have implemented several initiatives in order to present our Centres like a place that is open to all and not only dedicated to people with dementia. But it is still hard work. There is a bias towards the MCSP." Another stakeholder from Italy explains: "In the other district this has been a huge barrier. Another Centre with specific activities for old people was already present and we had problems with these people. There was a high resistance from the elders of both pre-existing Centres." In Poland, a wide variety of activities and social contacts emerged within and outside of the MCSP. However, it was sometimes difficult to connect with for instance other day care participants. A stakeholder explains: "Day care is usually for people with mental or physical disabilities. Day care participants are usually passive, not interested in many activities." There was also a problem with stereotypes and beliefs among day care users and professionals at the beginning: "They used to be a very close group." In the UK, stakeholders mentioned that while there is some integration with for instance people from local senior organizations, there is also still a lack of awareness about the existence of the Meeting Centers. Another stakeholder mentioned that there is still a stigma that has to do with dementia that prevents people from integrating.

\section{Continuation phase}

On a micro level, all countries valued family caregiver involvement and found that this clearly facilitated the implementation of MCSP. A stakeholder from Italy explained: "Often they need some respite time from their loved ones because the burden is so heavy." A stakeholder from Poland mentioned this as a barrier: "There was little engagement of carers participating in the MCSP programme. Only about 5 from 16 attended the support groups and informative meetings." In the UK, a stakeholder clearly stated that "Without them, many people with dementia would not attend the Meeting Centres." However, it appeared sometimes difficult to involve caregivers, primarily in Italy and Poland.

In Italy and the UK, the presence of a national dementia strategy facilitated the implementation, but there were also barriers. In Italy, some stakeholders mentioned that there is a national plan for Alzheimer's disease, but the MCSP still need to be implemented in the national plan. A stakeholder from Poland explained: "There are trends in support for elderly people but the regulations are not adjusted to support people with dementia and their families." A stakeholder from the UK: "the MCSP falls in between health and social care. People who use MCSP do not fit into an obvious category for personal budgets." According to regulations, a person with dementia is not eligible for a personal budget if they have mild to moderate dementia, and thus, lower support needs.

\section{Discussion}

\section{Facilitators and barriers at micro level}

Our results show that on a micro level, suitable staff and management, the presence of a professional project manager and the fact that the MCSP is attuned to the needs and wishes of people with dementia and informal caregivers facilitated the implementation in all countries in both the execution and continuation phase.

Social integration of the participants with dementia with other programs at the location of the MCSP was difficult in all countries during the execution phase of the implementation, but proved to facilitate implementation during the continuation phase.

A barrier mentioned by all countries during the continuation phase of implementation was that when the person with dementia needed more advanced care, there was a lack of capacity in other facilities. This was due to long waiting lists for long term care.

The main differences between countries for facilitators and barriers of the implementation during the continuation phase were related to: communication with potential referrers, discharge criteria for the MCSP and structuralfinancing. 


\section{Facilitators and barriers at meso level}

On a meso level, setting up an inter-organizational collaboration network, including clear protocols facilitated the implementation in Poland and the UK during the execution phase. However, setting up a network in Italy proved to be difficult. Furthermore, active PR facilitated the implementation in Poland and the UK during the continuation phase, while in Italy, good PR was considered less feasible because of time limits of the researchers. This was experienced as a barrier to the implementation. The image of the MCSP was positively perceived by all three countries during the continuation phase, especially in Poland and the UK.

\section{Facilitators and barriers at macro level}

On a macro level, differences between countries emerged with respect to support by national organizations in the execution phase. While Poland experienced a lot of support by national organizations that facilitated the implementation, the UK as well as Italy received less support by national organizations. The presence of a national dementia strategy facilitated the implementation of the MCSP during the continuation phase in Italy and the UK. However, in Poland and the UK the concept of MCSP did not exactly fit into a category for personal budgets (most people would not be eligible). In Italy, MCSP was not yet implemented in the existing national plans, which impeded the implementation.

\section{Comparison with Dutch experiences with implementation of MCSP}

The facilitators and barriers that were found in this study are comparable to those found in the implementation study of the MCSP in the Netherlands (Meiland et al., 2005).

Motivated staff and management, a dedicated project leader, financial support, continuous PR activities, perceived value of the MCSP program by other organizations, and cooperation between organizations were the main facilitators of the implementation of MCSP in the Netherlands. However, not all these factors were found to be facilitating in the three countries in this study. For instance, in the UK there were difficulties with communication between the MCSP and referrers and in Italy insufficient PR activities were done and it was difficult to set up a proper collaboration network for MCSP. The main barriers to the implementation of MCSP in the Netherlands were: a lack of capacity in other facilities for more advanced care, waiting lists for the MCSP and referrers who are not familiar with the added value of MCSP (including social integration and carer support). These barriers also influenced the implementation of the MCSP in the three countries. However, they differed in the extent to which they were experienced. Waiting lists were predominantly an issue in Poland and Italy. In the UK (initially) there was more of a problem with referrers who were not familiar with the added value of the MCSP, and unclear discharge criteria. Based on the Dutch experiences, a checklist was used to inventory potential facilitators and barriers and to discuss possible effective implementation strategies. This was part of a step-by-step implementation procedure, that appeared feasible and supportive (Mangiaracina et al., 2017). It helped stakeholders to develop effective strategies in their own context and region. Multi-targeted implementation strategies are recommended because varying facilitators and barriers were found in different phases of implementation and on different levels. Also, differences were found between countries and regions. Strategies should therefore be well attuned to these contextual differences. This worked well in the MEETINGDEM project and resulted, until now, in the implementation of a total of thirteen MCs in the participating countries (two or more MC's per country).

\section{Comparison with the implementation of other psychosocial interventions}

The results of this study are also comparable to other process evaluations into the implementation or improvement of psychosocial support interventions for people with dementia and their informal caregivers. Factors that were previously found to positively influence the implementation of psychosocial interventions are for instance the presence of motivated staff, strong management, good collaboration with other dementia care organizations and the extent to which the support program enables social participation (Dopp et al., 2013; Van Mierlo et al., 2014; Boersma et al., 2015; Van Haeften-van Dijk et al., 2015), the perceived value of the support program by other organizations (Minkman et al., 2009) and the involvement of family caregivers (Lawrence et al., 2012).

The strength of this study is that the implementation of MCSP was studied and compared across three very different European countries, regarding geography, culture and care system, at the same time. Although only a few number of Meeting Center's were involved per country, to date, no other studies have previously carried out such a comparison of implementation of a comprehensive support program between countries. Though 
different interviewers collected the data in each country, facilitators and barriers were inventoried by means of a standardized checklist, which contributes to the reliability of the data.

There were a few limitations to this study. In each country, the process evaluation was conducted for only one implemented Meeting Center (except for Italy where two Meeting Centers were subject of this process evaluation because they were implemented in about the same period). In addition to the country specific facilitators and barriers, regional differences within a country can also influence the implementation, such as law regulations and the organization of the dementia care network. Another limitation is that due to time limits the continuation phase of implementation was investigated a year after the opening of the Meeting Centers. This is somewhat early to make a clear assessment what factors influenced the implementation during the continuation phase. Furthermore, though the implementation of the MCSP was supported by a large research project (which was a facilitator), and led by highly motivated researchers, so-called "early adopters" (Rogers, 2003), these facilitating circumstances will probably not be the same in future implementations of MCSP. Because of these reasons, the results of the study need to be treated with caution with respect to the generalizability. However, future MCSP's that will be set up can benefit from the developed country specific implementation plans in the MEETINGDEM project and the practical implementation guides. The last will be developed based on the results of this study, as well as the previous study by Mangiaracinaetal. (2017).

Last, the type and number of interviewed stakeholders varied across the countries, and also the questions that were asked. These were related to stakeholders expertise and involvement in the implementation process. Therefore, we could not provide a precise summary of how often factors were experienced as a facilitator or barrier. We could however, provide a variety of experienced facilitators and barriers, as we intended.

This study demonstrates that it is feasible to adaptively implement the MCSP in different European countries and provides insight into which facilitators and barriers influenced theimplementation. These findings are relevant for research and practice: they can feed the development of and research into successful implementation strategies also for other psychosocial interventions. Though we have used the Theoretical Model of Adaptive Implementation in this study, other implementation models, such as the RE-AIM model (Glasgow et al., 1999; 2001) and the Implementation of Change model (Grol et al., 2005) are also useful frameworks to plan and study successful implementation strategies. Overall, setting up more Meeting Centers across Europe will contribute potentially to better individualized care for people with dementia and their informal caregivers, as MCSP give the target group a voice in both the content and execution of the program offered. This is expected to positively impact their quality of life and possibly delay institutionalization. The results of our study contribute to an understanding of the different facilitators and barriers that can be experienced during the implementation of psychosocial support programs in different European countries. Our study informs further implementation and dissemination of MCSP in the three target countries as well as in other countries in (and beyond) Europe and may also serve as an example for the dissemination and implementation of other psychosocial support interventions across Europe. Continuing research on the implementation of effective and combined care support is important in order to provide people with dementia and their caregivers with adequate support now and in the future.

\section{Conflict of interest declaration}

None.

\section{Description of authors' roles}

LvM, RC, SE, DB, FLS, GG, CS, EF, DS, KU, JR, RMD, and FJM were all involved in the design of the study and the writing the final version of the manuscript. LvM and FJM were involved in the analysis of the checklist data and the writing of the draft.

\section{Acknowledgments}

None.

\section{References}

Barbour, R. S. (1999). The case for combining qualitative and quantitative approaches in Health Services Research. Journal of Health Services Research Policy, 4, 39-43.

Boersma, P., Van Weert, J. C., Lakerveld, J. and Dröes, R.M.(2015). The art of successfulimplementation of psychosocial interventions in residential dementia care: a systematic review of the literature based on the RE-AIM framework.

Brodaty, H., Green, A. and Koschera, A. (2003). Meta-analysis of psychosocial interventions for caregivers of people with dementia. Journal of the American Geriatric Society, 51, 657-664. 
Dopp, C. M., Graff, M.J., Rikkert,M.G.M.O., Nijhuis van der Sanden, M.W.G. and Vernooij-Dassen, M.J.F.J.(2013). Determinants for the effectiveness of implementing an occupational therapy intervention in routine dementia care. Implementation Science, 8 , 131.

Dröes, R. M. et al. (2017). Comparison of the adaptive implementation and evaluation of the Meeting Centers Support Program for peoplewith dementia and their family carers in Europe; study protocol of the MEETINGDEM project. BioMed Central Geriatrics, 17, 79.

Dröes, R. M., Breebaart, E., Meiland, F. J. M., Van Tilburg, W. and Mellenbergh, G. J. (2004a). Effect of meeting centers support program on feeling of competence of family caregivers and delay of institutionalization of people with dementia. Aging \& MentalHealth, 8, 201-211.

Dröes, R. M., Breebaart, E., Van Tilburg, W. and Mellenbergh, G. J. (2000). The effect of integrated family support versus day care only on behavior and mood of patients with dementia. International Psychogeriatrics, 12, 99-116.

Dröes, R. M., Meiland, F. J. M., De Lange, J., Vernooij-Dassen, M. and Van Tilburg, W. (2003). The meeting centers support program; an effective way of supporting people with dementia who live at home and their carers. Dementia. The International Journal of Social Research and Practice, 2, 421-438.

Dröes, R. M., Meiland, F. J. M., Schmitz, M. and Van Tilburg, W. (2004b). Effect of combined support for people with dementia and carers versus regular day care on behavior and mood of persons with dementia: results from a multi-center implementation study. International Journal of Geriatric Psychiatry, 19, 1-12.

Dröes, R. M., Meiland, F. J. M., Schmitz, M. and Van Tilburg, W.(2011). An evaluation of the meeting centers support program among persons with dementia and their carers. Nonpharmacological Therapies in Dementia, 2, 19-39.

Glasgow, R. E., McKay, H. G., Piette, J. D. and

Reynolds, K. D. (2001). The RE-AIM framework for evaluatinginterventions: whatcanittellusabout approaches to chronic illness management? Patient Education and Counseling, 44, 119-127.

Glasgow, R. E., Vogt, T. M. and Boles, S. M. (1999). Evaluating the public health impact of health promotion interventions: the RE-AIM framework. American Journal of Public Health, 89, 1322-1327.

Grol, R. and Wensing, M. (2004). What drives change? Barriers to and incentives for achieving evidence-based practice. The Medical Journal of Australia, 180, S57-S60.

Grol, R., Wensing, M. and Eccles, M. (2005). Improving Patient Care; The Implementation of Change in Clinical Practice. Oxford: Elsevier.
Lawrence, V., Fossey, J., Ballard, C., Moniz-Cook, E. and Murray, J. (2012). Improving quality of life for people with dementia in care homes: making psychosocial interventions work. British Journal of Psychiatry, 201, 344-351.

Mangiaracina, F. et al. (2017). Not re-inventing the wheel: the adaptive implementation of the meeting centres support programme in four European countries. Aging and Mental Health, 21, 40-48.

Meiland, F. J. M., Dröes, R. M., De Lange, J. and Vernooij-Dassen, M. (2004). Development of a theoretical model for tracing facilitators and barriers in adaptive implementation of innovative practices in dementia care. Archives of Geriatrics and Gerontology, 9, 279-290.

Meiland, F. J. M., Dröes, R. M., De Lange, J. and VernooijDassen, M. (2005). Facilitators and barriers in the implementation of the meeting centers model for people with dementia and their carers. Health Policy, 71, 243-253.

Minkman, M. M., Ligthart, S. A. and Huijsman, R. (2009). Integrated dementia care in The Netherlands: a multiple case study of case management programs. Health and Social Care in the Community, 17, 485-494.

Moniz-Cook, E., Vernooij-Dassen, M., Woods, B. and Orrell, M. (2011). Psychosocial interventions in dementia care research: the INTERDEM manifesto. Aging \& Mental Health, 15, 283-290.

Olazarán, J.et al. (2010). Nonpharmacological therapies in Alzheimer's disease: a systematic review of efficacy. Dementia and Geriatric Cognitive Disorders, 30, 161-178.

Rogers, E. M. (2003). Diffusion of Innovation. 5th edn. New York: Free Press.

Smits, C.H., de Lange, J., Dröes, R.M., Meiland, F.J.M., Vernooij-Dassen, M. and Pot, A.M. (2007). Effects of combined intervention programs for people with dementia living at home and their caregivers: a systematic review. International Journal of Geriatric Psychiatry, 22, 1181-1193.

Van Haeften-van Dijk, A. M., Meiland, F. J. M., Van Mierlo, L. D. and Droës, R. M. (2015). Transforming nursing home-based day care for people with dementia into socially integrated community day care: process analysis of the transition of six day care centres. International Journal of Nursing Studies, 52, 1310-1322.

Van Mierlo, L. D., Meiland, F. J., Van Hout, H. P.J. and Dröes, R. M. (2014). Towards personalized integrated dementia care: a qualitative study into the implementation ofdifferentmodels of case management.BMCGeriatrics, 14,84 .

Vernooij-Dassen, M. and Moniz-Cook, E. (2014). Raising the standard of applied dementia care research: addressing theimplementation error. Aging \& Mental Health, 12, 1-6. 